\title{
CLINICAL CHARACTERISTICS OF PATIENTS WITH CYCLIC VOMITING SYNDROME IN SOUTHERN IRAN
}

\author{
M. Hadibarhaghtalab ${ }^{1}$, N. Hadibarhaghtalab ${ }^{2}$, A. Manafi ${ }^{1}$ \\ ${ }^{1}$ Student Research Committee of Fasa University of Medical Sciences, ${ }^{2}$ Pediatrician of Shiraz University of \\ Medical Science, Shiraz, Iran
}

Background: Cyclic vomiting syndrome (CVS) is characterized by recurrent episodes of intense nausea and vomiting interpreted with symptom free period. As data regarding the pattern of disease in our region is scarce, we performed this study to investigate the pattern of pediatric CVS in southern Iran.

Method: This was a retrospective analysis of 43 patients admitted to Nemazee hospital from 2000 to 2010 with diagnosis of cyclic vomiting according to the American Society for Pediatric Gastroenterology,Consensus criteria. The patients' medical charts were reviewed and the demographic, epidemiologic, laboratory, data were recruited using a standard questionnaire and further analyzed.

Result: Overall we included 43 patients with CVS among whom there were $26(60.5 \%)$ girls and 17 (39.5\%) boys. The mean age at first attack was found to be $6.2 \pm 3.7$ years (range 2 month to 15 years). Intractable vomiting was found in $16(37 \%)$ patients and it was more common than any other type of vomiting. 10 patients $(23.3 \%)$ had family history of migraine among which there were 8 female. The most clinical complaint was found to be abdominal pain in $32(74.4 \%)$ patients followed by headache in $19(44.2 \%)$.The most common trigger factor was upper respiratory tract infection detected in $13(30.2 \%)$ patients followed by urinary infection in $12(27.9 \%)$. The most associated disorder was abdominal migraine in $5(11.6 \%)$ patients.

Conclusion: The presentation of CVS shares many similarities with migraine and thus the treatment options could be potentially similar. 